\title{
Über die $\boldsymbol{N}$-Serie der Röntgenspektren.
}

\author{
Von V. Dolejšek.
}

Mit zwei Abbildungen. (Eingegangen am 9. Mai 1922.)

Vor kurzem ist ein vervollkommneter Spektrograph im Lunder Institute konstruiert und in der ZS. f. Phys. beschrieben worden 1). Diese vervollkommnete Apparatur wurde mir von Herrn Prof. Siegbahn in freundlichster Weise zur Verfügung gestellt und dadurch wurde die vorliegende Untersuchung ermöglicht. Außerdem hat zur Erzielung der Resultate hauptsächlich beigetragen, daß ich jetzt eine neve Art ${ }^{2}$ ) von photographischen Platten benatzt habe, die für die in dieser Arbeit in Betracht kommenden langwelligen Strahlen besonders empfindlich waren.

Untersucht wurden die Elemente Uran, Thorium und $W$ ismut. Sowohl bei den Anfnahmen wie bei der Identitizierung der Linien stellten sich mannigfache Hindernisse in den $\mathrm{Weg}$. Die wesentlichsten sind:

1. starke Absorption der Strahlen in der Spaltbedeckung;

2. Uberlagerung der $\mathbb{M}$-Linien in höheren Orduungen;

3. Schwärzung der Platte durch die diffuse Strahlung und durch Licht von der Antikathode;

4. eigene Breite der Linien (welche speziell bei den großen Glanzwinkein sich bemerkbar macht) and damit zusammenhängende Einbuße an Intensität.

Die günstigsten Expositionszeiten waren nicht länger als eine balbe bis eine ganze Stunde. Längere Aufuahmen brachten keinen Vorteil wegen der allgemeinen Schwärzung der Platte. Deswegen habe ich auch bei jeder Aufnahme nur kleine Winkelgebiete (gewöhnlich $1^{0}$ ) aufgenommen. An den Stellen, wo Linien gefunden wurden, sind mehrere Aufnahmen (gewöhnlich mit um 30' verschobenem Kristall) genommen worden.

Am Anfang, wo ich mit sehr schmalem Spalt gearbeitet habe ( 0,02 bis $0,03 \mathrm{~mm})$, habe ich trotz aller Versuche nur Linien bekommen, welche sich als höhere Ordnungen der $M$-Linien erwiesen haben. Im allgemeinen lassen sich die Linien höherer Ordnung ${ }^{3}$ ) in diesem Falle

1) M. Siegbahn, ZS. f. Phys. 9, 68, 1922.

2) „Eclipse"-Imperialplatten.

$\left.{ }^{3}\right)$ Hauptsächlich sind gemeint die $M$-Linien des betreffenden Elementes. 
uicht vermeiden, weil man, um die $N$-Linien zu erhalten, mit großer Energie arbeiten muß. Um dies zu erreichen, kann man nicht mit Spannungen arbeiten, die kleiner sind als die kritische Erregungsspannung der $M$-Reihe. $E s$ ist leicht einzusehen, daß gerade die Intensität solcher Linien, welche schon bei sehr schmalem Spalt gauz breit und diffus sind, und welche also ein endliches Frequenzbereich darstellen, in hohem Maße von der Spaltbreite abhängt. Weitere Aufnahmen sind dann mit einer Spaltbreite von etwa $0,1 \mathrm{~mm}$ gemacht worden. Die von dem Antikathodenmaterial herrührenden Linien waren leicht durch Ausweehslung des Antikathodenmaterials zu eliminieren. Alle erhaltenen Linien sind dann weiter eingehend geprüft worden, um Linien höherer Ordnung auszuscheiden, und zwar in folgender Weise:

1. durch Absorptionsversuche ${ }^{1}$ ),

2. dureb Vergleichung der Aufnahmen, die mit verschiedenen Kristallen erbalten sind (Gips, Zucker).

Nach dem ersten Verfahren wurde Aluminiumfolie von $0,007 \mathrm{~mm}$ Dicke oder dünnes schwarzes Papier zwischengeschaltet. Mit der Folie wurde die obere Hälfte der Platte überdeckt, so daß man auf der Platte gleichzeitig die ungeschwächte Linie und die Linie nach dem Durchgang durch die Folie bekam. Bei den Wellenlängen bis $9 \AA$. .-E. kann man mit obiger Al-Folie gut auskommen, weil sie die Wellenlängen oberhalb $6 \AA$ A.-E. $(P \cdot K \alpha)$ vollkommen absorbiert, dagegen die Wellenlängen bis etwa 5 Å.-E. merkbar durchläßt. Bei den untersuchten längeren Wellen, wo die Werte der $M$-Linien etwa 5 bis 6 Ä.-E. sein können (zerstäubtes Wolfram aus der Glühspirale), ist schwarzes Papier benutzt worden, von welchem z. B. die Na-K $\alpha$-Linie (11 $\AA$.-E.) volkommen absorbiert wurde. In zweifelhaften Fällen habe ich außerdem an den betreffenden Stellen in ersten Ordnung gesucht, ob dort vielleicht eine bisher unbekannte $M$-Linie oder irgend eine andere Linie liegen könnte. Zu der weiteren Entscheidung hat außerdem das verschiedene Reflexionsvermögen vou Gips und Zuckerkristall für verschiedene $W$ ellenlängen nnd Ordnungen viel beigetragen. Wie aus einigen Kontrollversuchen hervorging, werden im allgemeinen von dem Zuckerkristall die Linien in höherer Ordnung verhältnismäßig viel intensiver reflektiert als die Linien erster Ordnung, während beim Gipskristall das Umgekehrte der Fall ist. Doch ist es gelungen, auch nit dem Zuckerkristall die meisten der Linien zu erhalten. Dieser

1) Dieses Verfahren ist schon früher von Coster benutzt worden (Phil. Mag. Juni 1922). 
Sachverhalt gibt ein geeignetes Mittel, um za entscheiden, ob eine Linie in erster Ordnung auftritt oder nicht. Tabelle 1, 2, 3 geben die Werte der gefundenen Linien bei $\mathrm{U}$, Th and $\mathrm{Bi}$, welche wahrscheinlich der $N$-Serie angehören.

Tabelle 1. Uran 92.

\begin{tabular}{|c|c|c|c|c|c|}
\hline Platte & Linie & $\varphi$ & $\varphi$ & $\lambda$ & $\nu / R$ \\
\hline $\begin{array}{l}103 \\
102\end{array}$ & $N_{7} P_{1}$ & $\begin{array}{l}34^{0} 32,2^{\prime} \\
3433,1\end{array}$ & $34^{0} 32,6^{\prime}$ & 8594 & 105,90 \\
\hline $\begin{array}{l}101 \\
102\end{array}$ & $N_{7} P_{2}$ & $\begin{array}{ll}35 & 01,5 \\
35 & 02,5\end{array}$ & 3502,0 & 8700 & 104,74 \\
\hline $\begin{array}{r}18 \\
19 \\
20 \\
21 \\
103\end{array}$ & $N_{6} O_{2}$ & $\begin{array}{ll}40 & 37 \\
40 & 37,4 \\
40 & 36 \\
40 & 36,6 \\
40 & 36\end{array}$ & $40 \quad 36,6$ & 9876 & 92,28 \\
\hline $\begin{array}{r}20 \\
21 \\
103\end{array}$ & $N_{7} O_{3}$ & $\begin{array}{ll}41 & 40,5 \\
41 & 37,6 \\
41 & 39\end{array}$ & 4138 & 10080 & 90,40 \\
\hline $\begin{array}{l}107 \\
108 \\
110\end{array}$ & $\mathrm{~N}_{5} \mathrm{O}_{2}$ & $\begin{array}{ll}56 & 57,2 \\
56 & 56,3 \\
56 & 56,3\end{array}$ & $56 \quad 56,7$ & 12702 & 71,74 \\
\hline $\begin{array}{l}107 \\
108 \\
110\end{array}$ & $\lambda_{5} O_{1}$ & $\begin{array}{ll}57 & 29,0 \\
57 & 27,0 \\
57 & 28,2\end{array}$ & 5728,1 & 12777 & 71,32 \\
\hline $\left.\begin{array}{l}107 \\
108 \\
110\end{array}\right\}$ & $X_{6} O_{5}$ & $\begin{array}{ll}58 & 02,9 \\
58 & 00,1\end{array}$ & 5801,5 & 12856 & 70,88 \\
\hline
\end{tabular}

Tabelle 2. Thor 90.

\begin{tabular}{|c|c|c|c|c|c|}
\hline Platte & Linie & $\varphi$ & $\varphi$ & $i$ & $\nu R$ \\
\hline $\begin{array}{l}57 \\
58\end{array}$ & $I_{7} P_{1}$ & $\begin{array}{l}26^{0} 07^{\prime} \\
26 \quad 08,4\end{array}$ & $26^{0} 07, \pi^{\prime}$ & 9310 & 97,88 \\
\hline $\begin{array}{l}57 \\
58\end{array}$ & $N_{7} P_{2}$ & $\begin{array}{ll}26 & 28,0 \\
26 & 29,9\end{array}$ & 2629 & 9427 & 96,67 \\
\hline $\begin{array}{l}37 a \\
37 b \\
40\end{array}$ & $\mathrm{~N}_{7} \mathrm{O}_{3}$ & $\begin{array}{ll}49 & 04 \\
49 & 03,5 \\
49 & 04,6\end{array}$ & $49 \quad 04,0$ & 11462 & 79,50 \\
\hline $\begin{array}{l}37 \mathrm{a} \\
37 \mathrm{~b} \\
38 \\
40 \\
44\end{array}$ & $\mathrm{~N}_{6} \mathrm{O}_{2}$ & $\begin{array}{ll}49 & 32,6 \\
49 & 31,4 \\
49 & 32,0 \\
49 & 33,8 \\
49 & 32,8\end{array}$ & $49 \quad 32,4$ & 11542 & 78,86 \\
\hline 46 & $N_{5} P_{3}$ & $59 \quad 53,5$ & $59 \quad 53,5$ & 13111 & 69,50 \\
\hline 46 & $N_{5} O_{2}$ & $60 \quad 25,3$ & 6025,3 & 13181 & 69,14 \\
\hline 46 & $N_{5} O_{1}$ & 6053,5 & 6053,5 & 13255 & 68,67 \\
\hline
\end{tabular}


Tabelle 3. Bi 83.

\begin{tabular}{|c|c|c|c|c|c|}
\hline Platte & Linie & $\varphi$ & $\varphi$ & $\lambda$ & $\nu / R$ \\
\hline $\begin{array}{l}112 \\
112\end{array}$ & $\frac{N_{7}}{\Lambda_{7}^{*} P_{1}}$ & $\begin{array}{l}59^{0} 23,6^{\prime} \\
60 \quad 06,5\end{array}$ & $\begin{array}{l}59^{0} 23,6^{\prime} \\
60 \quad 06,5\end{array}$ & $\begin{array}{l}13040 \\
13140\end{array}$ & $\begin{array}{l}69,9 \\
69,4\end{array}$ \\
\hline
\end{tabular}

Für die Identifizierung der gefundenen Linien mit den $N$-Linien spricht auch der Vergleich mit der Theorie. Bekanntlich ist es in der letzten Zeit gelungen, für die Röntgenspektren der schwersten Elemente ein cinfaches und sehr befriedigendes Schema aufzustellen ${ }^{1}$ ), in welches die meisten der gemessenen Linien im $K$-, $L$ - und $M$-Gebiet eingereiht werden können. Für die Elemente in der Umgebang des Edelgases Niton (86) hat man in Ubereinstimmung mit Bohrs Theorie nach diesem Schema sieben $N$-, fünf $O$ - und drei $P$-Niveaus. In Fig, 1

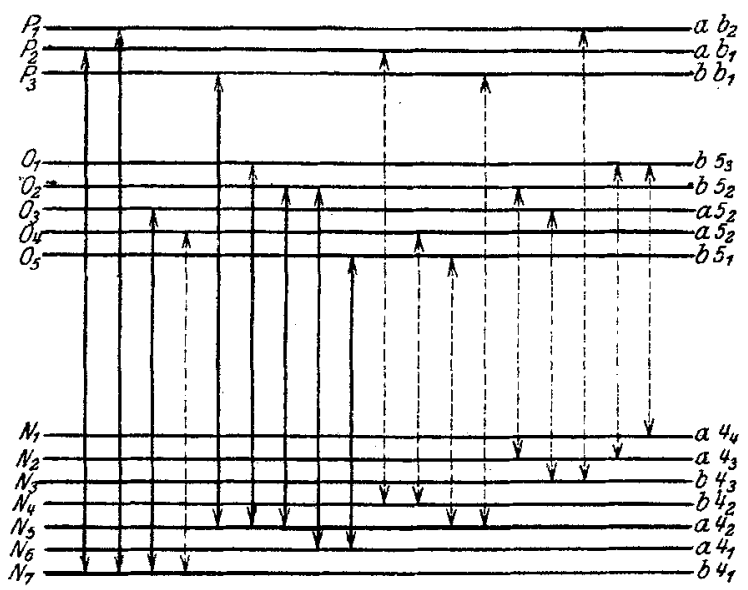

Fig. 1.

sind die Niveaus eingetragen. Jedes Niveau ist charakterisiert durch ein Quantensymbol der Form $n_{k}$ ( $n$ bedeutet die totale, $k$ die azimutale Quantenzahl). Weiter steht bei jedem Niveau der Buchstabe $a$ oder $b$. Die Utbergänge sind nach Coster den folgenden Bedingungen unterworfen: 1. die azimntale Quantenzahl soll sich um eine Einheit ändern oder sie soll konstant bleiben. 2. Die Ubergänge finden nur statt von einem $a$-Nivean nach einem $b$-Niveau oder umgekehrt. (Nicht von $a$ nach $a$ oder von $b$ nach $b$.)

1) D. Coster, ZS. f. Phys. 5, 139, 191 und 6, 185, 1y21; Arch. Néerl. (3 A) 6, 76, 1922, Heft 1; Phil. Mag. Juni 1922. Vgl. auch: A. Smekal, ZS. f. Phys. 5, 91, 121, 1921. G. Wentzel, ZS. f. Phys. 6, 84, 1921 und 8, $85,1921$. 
Alle tinien im $N$-Gebiet, welche nach diesem Schema möglich sind, wurden in die Fig. 1 eingetragen. Durch die gezogenen Iinien sind solche Utbergänge, welche mit den Werten der gefundenen Linien übereinstimmen, dargestellt. In Tabelle 4,5,6 sind die Frequenzwerte Tabelle 4. U 92.

\begin{tabular}{|c|c|c|c|c|}
\hline Linie & $\nu / R$ gem & $\nu / R$ ber. & Kombination & \\
\hline$N_{7} P_{1}$ & 105,9 & 105,5 & $\begin{array}{c}L_{1}-\beta_{6} \\
1264,2-1158,7\end{array}$ & $\begin{array}{c}P_{1} P_{2} \text {-Niveau bisher } \\
\text { nicht getrennt. }\end{array}$ \\
\hline$N_{7} P_{2}$ & 104,7 & & & \\
\hline$\Lambda_{6}^{+} O_{2}$ & 92,3 & 91,0 & $\begin{array}{c}L_{3}-\gamma_{2}-\left(L_{2}-\gamma_{0}\right) \\
1603,4-1507,8-(1542,4-1537,8)\end{array}$ & \\
\hline $\mathrm{N}_{7} \mathrm{O}_{3}$ & 90,4 & 90,5 & $\begin{array}{c}L_{1}-\beta_{6}-\left(L_{3}-\gamma_{4}\right) \\
1264,2-1158,7-(1603,4-1588)\end{array}$ & \\
\hline$N_{5} P_{3}$ & - & 77,6 & $\frac{L_{3}-\gamma_{3}-P_{3}}{1604,0-1526,4-P_{3}}$ & $\begin{array}{l}\text { Fällt m. d. } 3 T \text {-Linie zusam- } \\
\text { men; gemessen bei Th. }\end{array}$ \\
\hline $\mathrm{N}_{5} \mathrm{O}_{2}$ & 71,8 & 72,4 & $\begin{array}{c}L_{3}-\gamma_{3}-\left(L_{2}-\gamma_{6}\right) \\
1603,4-1526,4-(1542,4-1537,8)\end{array}$ & \\
\hline$N_{5} O_{1}$ & 71,3 & 71,3 & $\begin{array}{c}L_{3}-\gamma_{3}-\left(L_{1}-\beta_{5}\right) \\
1603,4-1526,4-(1264,2-1258,4)\end{array}$ & \\
\hline$N_{6} O_{b}$ & 70,9 & 69,5 & $\begin{array}{c}L_{3}-\gamma_{2}-\left(L_{1}-\beta_{7}\right) \\
1603,4-1507,8-(1264,2-1238,1)\end{array}$ & \\
\hline
\end{tabular}

Tabelle 5. Tb 90.

\begin{tabular}{|c|c|c|c|c|}
\hline Linie & $v / R$ gem. & $\psi / R$ ber: & Kombination & \\
\hline$N_{7} P_{1}$ & 97,9 & 97,9 & $\begin{array}{c}L_{1}-\beta_{6} \\
1200,7-1102,8\end{array}$ & \\
\hline $\begin{array}{l}N_{7} P_{2} \\
N_{6} O_{2}\end{array}$ & $\begin{array}{l}96,9 \\
78,9\end{array}$ & 76,5 & $\begin{aligned} L_{3}-\gamma_{2} & -\left(L_{2}-\gamma_{6}\right) \\
1509,7-1428 & -(1451,5-1446,2)\end{aligned}$ & \\
\hline$N_{7} O_{3}$ & 79,5 & 79,5 & $\begin{array}{r}L_{1}-\beta_{6}-\left(L_{3}-\gamma_{4}\right) \\
1200,7-1102,8-(1509,7-1491)\end{array}$ & \\
\hline$N_{5} P_{3}$ & 69,5 & 73,7 & $\begin{aligned} L_{3}-\gamma_{3} & -P_{3} \\
1509,7-1436 & =P_{3}\end{aligned}$ & $P_{3}$ nicht abgezogen. \\
\hline$N_{5} O_{2}$ & 69,1 & 68,5 & $\begin{array}{l}-\left(L_{2}-\gamma_{0}\right) \\
-(1451,5-1446,2)\end{array}$ & \\
\hline$N_{5} O_{1}$ & 68,7 & 68,1 & $\begin{array}{c}L_{3}-\gamma_{3}-\left(L_{1}-\beta_{5}\right) \\
1509,7-1436-(1200,7-1194,9)\end{array}$ & \\
\hline$N_{6} O_{5}$ & - & 50 & $\begin{aligned} L_{3}-\gamma_{2} & -\left(L_{1}-\beta_{7}\right) \\
1509,7-1428 & -(1200,7-1177)\end{aligned}$ & $\left\{\begin{array}{l}\text { Zu langwellig; etwa } \\
15 \text { A.-E. gem. bei Ur. }\end{array}\right.$ \\
\hline
\end{tabular}

Tabelle 6. Bi 83.

\begin{tabular}{|c|c|c|c|}
\hline Eoinie & $\nu / R_{\text {gem }}$ & $v / R$ ber. & Kombination \\
\hline $\begin{array}{l}N_{7} P_{1} \\
P_{7} P_{2}\end{array}$ & $\begin{array}{l}69,9 \\
69,4\end{array}$ & 70,8 & $\begin{array}{c}L_{1}-\beta_{6} \\
989,8-919,0\end{array}$ \\
\hline
\end{tabular}


der gefundenen Linien mit den berechneten Werten zusammengestellt. In der ersten Spalte warde der betreffende Utbergang angegeben, in der zweiten Spalte die gemessene Frequenz, in der dritten Spalte die berechnete Frequenz. In der vierten Spalte ist angegeben, aus welchen Daten (mit Hilfe des oben erwähnten Schemas) die Frequenzen berechnet worden sind. Die Werte der Frequenzen der $L$-Linien wurden Costers Arbeiten entlehnt. Für die Werte der $L$-Grenzen sind eingesetzt die Werte, welche man aus den $L$-Linien und aus den von Stenström ${ }^{1}$ ) and Coster ${ }^{2}$ ) gemessenen $M$-Grenzen berechnen kann.

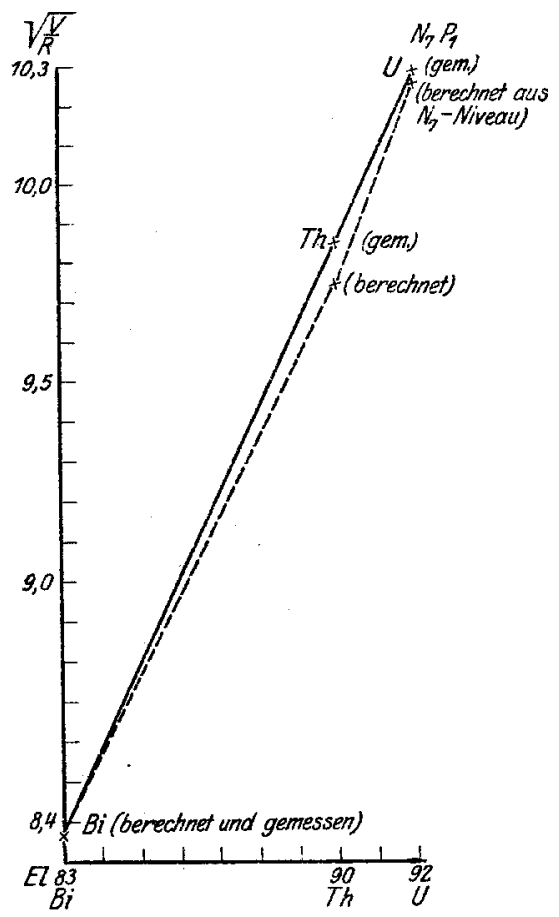

Fig. 2.

Dadurch ist größere Übereinstimmung erzielt, als mit den Werten von Duane und Patterson ${ }^{3}$ ). Das dies berechtigt ist, ist daraus za ersehen, daß die Linien im allgemeinen besser zu bestimmen sind als die Kanten. Ein Fehler in der Wellenlänge der $M$-Kante hat außerdem auf den fraglichen Frequenzwert einen kleinen Einfluß. Es sind dabei immer die zuverlässigsten Werte genommen. Die $L_{1}$-Grenze

1) Stenström, Diss. Lund 1919.

2) Coster, Phys. Rev. 19, 20, 1922.

3) Duane und Patterson, Proc. Nat. Ac. Washington 6, 509, 1920. 
ist berechnet aus $M_{1}$ und $L \alpha_{1}$, die $L_{2}$-Grenze aus $\mathbb{M}_{2}$ und $L \beta_{1}$, die $L_{3}$-Grenze ans $M_{3}$ and $L \beta_{3}$. Die Berechtigung dieses Verfahren kann man auch aus Fig. 2 schließen. Dortselbst wurde eingetragen die härteste Linie, die auch bei Wismut gemessen werden konnte, und welche etwa dieselbe Wellenlänge haben soll, als die (niemals gemessene) $N_{7}$-Grenze. Wie dort zu sehen ist, folgt diese Linie der Moseley-Frequenzbedingung (ebenso wie die korrigierten ausgerechneten $N_{7}$-Werte), die Werte dagegen, die aus den Duane und Pattersonschen $L$-Werten berechnet worden sind (gestrichelte Linie), zeigen Abweichungen.

In zwei Fällen war es unmöglich, die Linie, die bei einem Elemente gefunden wurde, bei dem anderen Elemente nachzaweisen. Bei $U$ bei der Linie $N_{5} P_{3}$ deswegen, weil an dieser Stelle eine $M$-Linie in höherer Ordnung erschien, bei Th bei der Linie $N_{6} O_{5}$, weil diese außerhalb des erreichbaren Gebietes liegt.

Wie aus den Tabellen ersichtlich, lassen sích alle Linien, welche gefunden worden sind, in das Schema einreihen. Die Übereinstimmung zwischen den gemessenen und berechneten Werten liegt in der Regel ganz innerhalb der Fehlergrenzen. Es soll hier aber bemerkt werden, da $B$ die Frequenz der Linie $N_{7} P_{1}$ sich nicht genau durch die Frequenzdifferenz $L_{1}-\beta_{6}$ vorstellen läßt, weil das $P_{1}$-Niveau bei $\mathrm{Ur}$ und $\mathrm{Th}$ nicht mehr ganz an der Peripherie des Atoms liegt. Daß keine Funkenlinien ${ }^{1}$ ) auftraten, trotzdem gerade in der $N$-Serie viele zu erwarten sind, darf nicht wandern, da die Linien, welche sich in das Diagramm einreihen lassen, schon sehr scbwach auf den Platten hervortraten und bekanntlich die Röntgenfunkenlinien im allgemeinen viel schwächer als die "Bogenlinien" sind.

Die in der Fig. 1 gestrichelten Utbergänge, welche man auch erwarten könnte, die aber bisher nicht wahrgenommen wurden, sind mit einer Ausnahme alle von so großen Wellenlängen, daß sie nicht gemessen werden können. Eine solche Ausnahme bildet die Linie $\mathrm{N}_{7} \mathrm{O}_{4}$, welche auf keiner Platte mit Sicherheit gezeigt werden konnte. Es soll hier noch darauf hingewiesen werden, daß, falls meine Identifizierung der Linien richtig ist, es hier zum ersten Male gelungen ist, die relativistischen Feinstrukturdubletts $P_{1}-P_{2}$ and $O_{1}-O_{2}$ experimentell aufzuspalten. Berechnet man aus den experimentell gefundenen Werten dieser Dubletts nach Som merfelds Formel die Absehirmungskonstanten, so bekommt man für das $P_{1}-P_{2}$-Dublett (Quantenzahlen $6_{2}$ und $6_{1}$ ) etwa 30 , und für das $O_{1}-O_{2}$-Dublett (Quantenzahlen $5_{3}$

1) Wentzel, Ann. d. Phys. 66, 437, 1921. 
and $5_{2}$ ) etwa 60 . Die erste Zahl scheint sehr vernünftig za sein, die zweite Zahl ist vielleicht etwas größer, als man erwarten möchte.

Da nur von zwei Elementen ein beträchtlicher Teil des N-Spektrums anfgenommen werden konnte, ist alles noch ein wenig unsicher; eine weitere Bestätigung bzw. Korrektion der vorliegenden Arbeit könnte aber eine genaue Untersuchung der $M$-Reihe bringen.

Im allgemeinen kann man sagen, daß die Übereinstimmung zwischen der theoretischen Erwartung und den experimentell gefundenen Tatsachen so gut ist, wie man nur hoffen konnte, und daß die gefandenen Linien eine weitere Bestätigung des Schemas geben.

Ich möchte dem Direktor des Lunder Instituts, Herrn Prof. Manne Siegbahn, anch an dieser Stelle für sein großes Interesse und seine wertvollen Ratschläge herzlichst danken.

Lund, Fysiska Institutionen, 2. Mai 1922. 